\title{
Global Symplectic Uncertainty Propagation on $\mathrm{SO}(3)$
}

\author{
Taeyoung Lee*†, Melvin Leok*, and N. Harris McClamroch ${ }^{\dagger}$
}

\begin{abstract}
This paper introduces a global uncertainty propagation scheme for the attitude dynamics of a rigid body, through a combination of numerical parametric uncertainty techniques, noncommutative harmonic analysis, and geometric numerical integration. This method is distinguished from prior approaches, as it allows one to consider probability densities that are global, and are not supported on only a single coordinate chart on the manifold. It propagates a global probability density through the full attitude dynamics, instead of replacing angular velocity dynamics with a gyro bias model. The use of Lie group variational integrators, that are symplectic and remain on the Lie group, as the underlying numerical propagator ensures that the advected probability densities respect the geometric properties of uncertainty propagation in Hamiltonian systems, which arise as consequence of the Gromov nonsqueezing theorem from symplectic geometry. We also describe how the global uncertainty propagation scheme can be applied to the problem of global attitude estimation.
\end{abstract}

\section{INTRODUCTION}

The attitude dynamics of a rigid body is a Hamiltonian flow on the special orthogonal group $\mathrm{SO}(3)$, but most current attitude uncertainty propagation schemes [1] do not properly take these characteristics into account. Typically, the attitude is represented by unit quaternions, which is problematic for global uncertainty propagation due to the ambiguity introduced by the double cover of $\mathrm{SO}(3)$ by the three-sphere $\mathbb{S}^{3}$ of unit quaternions. Since a covariance matrix for the quaternion vector is singular, reduced representations of the covariance matrix have been developed [2]. Furthermore, the dynamics are often simplified to kinematic equations by replacing the dynamics of the angular velocity with a gyro bias model, thereby ignoring uncertainties in the angular velocity [3]. As such, most existing techniques are only valid over time periods when the uncertainties are small.

This paper develops a global uncertainty propagation method for a rigid body by explicitly considering the characteristics of the Hamiltonian flow and the special orthogonal group, without implicitly assuming that the uncertainty is localized, nor that the uncertainty distribution is fully supported in a single coordinate chart on the manifold. We also explicitly consider uncertainties in the angular velocity dynamics, and we propagate a probability density on the

Taeyoung Lee, Mechanical and Aerospace Engineering, Florida Institute of Technology, Melbourne, FL 39201 taeyoung@fit.edu

Melvin Leok, Mathematics, Purdue University, West Lafayette, IN 47907 mleok@math. purdue.edu

N. Harris McClamroch, Aerospace Engineering, University of Michigan, Ann Arbor, MI 48109 nhm@umich.edu

* This research has been supported in part by NSF under grants DMS0504747, DMS-0714223, and DMS-0726263.

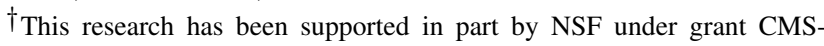
0555797 . tangent bundle of $\mathrm{SO}(3)$. This paper focuses on developing a computational approach for global uncertainty propagation that can be applied to aggressive attitude dynamics with large uncertainty. This is achieved through a synthesis of noncommutative harmonic analysis [4], numerical parametric uncertainty analysis techniques [5], [6], and geometric numerical integration [7].

Although it is not widely known in the engineering community, the theories of probability and stochastic processes on manifolds have been developed by theoretical statisticians [8], [9], [10]. Earlier works on attitude estimation on $\mathrm{SO}(3)$ include [11], where a probability density function is expressed using noncommutative harmonic analysis. This idea of using Fourier analysis on manifolds has been applied in [12], [13], [14], [15], and recently, results in [11] are extended to include the effects of process noise and sensor parameters in [16]. The use of noncommutative harmonic analysis allows a probability density function to be expressed globally on $\mathrm{SO}(3)$ without needing partition of unity methods on multiple coordinate charts.

The Liouville equation describes the evolution of a probability density function in the absence of external diffusion, and can be viewed as the deterministic analogue of the Fokker-Planck equation. When the flow advecting the probability density is Hamiltonian, the Liouville equation reduces to an ordinary differential equation [17]. Thus, a probability density function can be propagated using the flow map of the Hamiltonian system. In [18], an attitude estimation scheme is developed by linearizing the attitude dynamics along the mean obtained from the probability density function propagated by this property. However, nonlinearities of the flow imply that numerical methods for propagating uncertainties using linearization rapidly degrade in performance, unless frequent physical measurements are available [19]. It is therefore desirable to construct efficient numerical methods for solving the Liouville equation that describes the evolution of a probability density advected by a prescribed Hamiltonian flow.

Geometric numerical integration preserves the geometric properties of a dynamical system, such as invariants, symmetry, and reversibility [7]. We use a geometric numerical integrator for a rigid body, referred to as a Lie group variational integrator, that preserves the symplectic property of the Hamiltonian dynamics and the group structure of the configuration space [20], [21]. The Gromov nonsqueezing theorem [22] from symplectic geometry places fundamental limits on how the uncertainty of a Hamiltonian system evolves [23]. It is therefore essential that symplectic methods be used to propagate individual trajectories when analyzing 
uncertainty propagation in Hamiltonian systems.

The purpose of this paper is to present a computational method to propagate uncertainties under the attitude dynamics of a rigid body on $\mathrm{SO}(3)$. It is assumed that an initial probability density is prescribed. At a given future time, we backpropagate sample points in $\operatorname{TSO}(3)$ along the Hamiltonian flow of the rigid body dynamics, using a Lie group variational integrator. We use the advected probabilities to reconstruct the probability density at the given future time using noncommutative harmonic analysis on $\mathrm{SO}(3)$. This is in contrast to Monte Carlo methods, where the sample trajectories are used to compute statistical properties of the advected density. This also should be distinguished from numerical methods based on a specific realization of a stochastic Hamiltonian system [24], [25], [26].

The uncertainty propagation scheme we present has the following important features. A probability density function for the attitude dynamics of a rigid body is expressed globally on $\operatorname{TSO}(3)$. The uncertainty is propagated through the attitude dynamics on $\operatorname{TSO}(3)$. The approach ensures that the uncertainty propagation inherits the geometric properties of the time evolution of probability densities in Hamiltonian systems.

This paper is organized as follows. In Section II, we describe a specific attitude dynamics model, namely the 3D pendulum, and a Lie group variational integrator. An uncertainty propagation scheme is proposed in Section III, followed by computational results in Section IV. This development is also applied to global attitude estimation.

\section{Attitude Dynamics of A Rigid Body}

\section{A. 3D Pendulum}

A rigid $3 \mathrm{D}$ pendulum is a rigid body supported by a fixed, frictionless pivot, acted on by a uniform gravitational force [27]. The supporting pivot allows the pendulum three rotational degrees of freedom. This is a nontrivial example of a Hamiltonian system that evolves on a Lie group; this example is used in the subsequent development.

Two reference frames are introduced. An inertial reference frame has its origin at the pivot; the first two axes lie in the horizontal plane and the third axis is vertical in the direction of gravity. A reference frame fixed to the pendulum body is also introduced. The origin of this body-fixed frame is also located at the pivot. The configuration manifold is the special orthogonal group $\mathrm{SO}(3)$,

$$
\mathrm{SO}(3)=\left\{R \in \mathbb{R}^{3 \times 3} \mid R^{T} R=I_{3 \times 3}, \operatorname{det} R=1\right\},
$$

where the rotation matrix $R \in \mathrm{SO}(3)$ represents the linear transformation from the body-fixed frame to the inertial frame.

The dynamics of the 3D pendulum are given by the Euler rigid body equation that includes the moment due to gravity:

$$
J \dot{\Omega}=J \Omega \times \Omega+M,
$$

where the angular velocity in the body-fixed frame is denoted by $\Omega \in \mathbb{R}^{3}$, the inertia matrix is denoted by $J \in \mathbb{R}^{3 \times 3}$, and the vector $M \in \mathbb{R}^{3}$ represents the external moment due to the gravitational potential. It is given by $M=m g \rho \times R^{T} e_{3}$, where the vector $\rho \in \mathbb{R}^{3}$ represents the location of the center of mass in the body-fixed frame, and the constants $m$ and $g$ denote the mass of the pendulum and the gravitational acceleration, respectively. The kinematic equation is

$$
\dot{R}=R S(\Omega) \text {. }
$$

For a given vector $x \in \mathbb{R}^{3}$, the $3 \times 3$ skew-symmetric matrix $S(x)$ is defined so that $S(x) y=x \times y$ for all $y \in \mathbb{R}^{3}$.

There are two disjoint equilibria when the direction of gravity in the body fixed frame is collinear with the vector $\rho$; the hanging equilibrium when $R^{T} e_{3}=\rho /\|\rho\|$, and the inverted equilibrium when $R^{T} e_{3}=-\rho /\|\rho\|$. The 3D pendulum exhibits surprisingly rich and complicated attitude dynamics [19], and is therefore particularly appropriate for demonstrating the properties of our global attitude uncertainty propagation scheme.

\section{B. Lie Group Variational Integrator}

A Lie group variational integrator is a geometric numerical integrator for Hamiltonian systems on a Lie group. As group elements are updated according to the group operation, the group structure is preserved without the use of local charts, reprojection, or constraints. They are symplectic and momentum preserving, and they exhibit good energy behavior for an exponentially long time period [28].

The following Lie group variational integrator for the attitude dynamics of a rigid body was presented in [20], [21]:

$$
\begin{gathered}
h S\left(J \Omega_{k}+\frac{h}{2} M_{k}\right)=F_{k} J_{d}-J_{d} F_{k}^{T}, \\
R_{k+1}=R_{k} F_{k}, \\
J \Omega_{k+1}=F_{k}^{T} J \Omega_{k}+\frac{h}{2} F_{k}^{T} M_{k}+\frac{h}{2} M_{k+1},
\end{gathered}
$$

where the constant $h \in \mathbb{R}$ is the integration step size, and the subscript $k$ denotes the $k$-th integration step. The matrix $J_{d} \in \mathbb{R}^{3 \times 3}$ is a nonstandard inertia matrix given by $J_{d}=$ $\frac{1}{2} \operatorname{tr}[J] I_{3 \times 3}-J$, and $F_{k} \in \mathrm{SO}(3)$ is the relative attitude between integration steps. This integrator yields a discrete time flow map $\mathcal{F}:\left(R_{k}, \Omega_{k}\right) \mapsto\left(R_{k+1}, \Omega_{k+1}\right)$ by solving (3) to obtain $F_{k} \in \mathrm{SO}(3)$ and substituting it into (4) and (5) to obtain $R_{k+1}$ and $\Omega_{k+1}$.

As the rotation matrix is updated with the product of two rotation matrices in (4), the structure of $\mathrm{SO}(3)$ is preserved automatically. Since this integrator is derived from the discrete Hamilton's principle, the symplectic structure is conserved along the discrete-time flow. We use these discrete-time equations of motion to propagate the attitude dynamics.

\section{Global Symplectic UnCERTAinty PROPAGATION ON SO(3)}

Let a probability density function for the attitude and the angular velocity of a rigid body at time $t_{k}$ be denoted by $p_{k}(R, \Omega): \mathrm{SO}(3) \times \mathbb{R}^{3} \rightarrow \mathbb{R}$. In this section, we develop a computational method to propagate this density along the 
Hamiltonian attitude dynamics assuming that there is no process noise. Here we consider the full attitude dynamics on $\operatorname{TSO}(3) \simeq \mathrm{SO}(3) \times \mathbb{R}^{3}$, instead of simplifying it to a kinematics equation by using a gyro bias model [2], [3]. We represent the propagated probability density function globally on $\mathrm{SO}(3)$ using noncommutative harmonic analysis. Later, a new method for visualizing the attitude uncertainty on a two-sphere is also discussed.

\section{A. Symplectic Uncertainty Propagation for a Hamiltonian System}

In [17], it is shown that the probability density function is preserved along a Hamiltonian flow on Euclidean space. In this subsection, we generalize this result to a Hamiltonian system evolving on a general symplectic manifold.

Consider a Hamiltonian system on a $2 n$-dimensional symplectic manifold $(Q, \omega)$, where $Q$ is a $2 n$-dimensional manifold and $\omega: \mathrm{T} Q \times \mathrm{T} Q \rightarrow \mathbb{R}$ is a nondegenerate symplectic two-form on $Q$ [29]. The Liouville volume form $\mu:(\mathrm{T} Q)^{2 n} \rightarrow \mathbb{R}$ is defined as the $n$-fold wedge product of the symplectic two-form with itself, $\mu=\frac{(-1)^{n(n-1) / 2}}{n !} \omega \wedge$ $\cdots \wedge \omega$ ( $n$ times). In local coordinates, this corresponds to the usual notion of the volume element in Euclidean spaces. Let $\mathcal{L}_{X} \mu$ be the Lie derivative of the volume form $\mu$ along a vector field $X: Q \rightarrow \mathrm{T} Q$. The divergence of a vector field $X$ on $Q$ is defined as $\mathcal{L}_{X} \mu=\operatorname{div}_{\mu}(X) \mu$. Thus, the divergence $\operatorname{div}_{\mu}(X)$ represents the rate of change of a unit volume along the vector field $X$.

In [4], it is shown that the Fokker-Planck equation for a dynamical system on a manifold in the absence of diffusion terms can be written as,

$$
\frac{\partial p}{\partial t}+\operatorname{div}_{\mu}(p X)=0
$$

Note that this has the same structure as the Euler equation for the density of compressible fluids. The existence and uniqueness of the solution of the equations of motion provides a property analogous to mass conservation in fluid dynamics.

The time derivative of the probability density function is given by

$$
\frac{d}{d t} p=\frac{\partial p}{\partial t}+\mathcal{L}_{X} p
$$

Using the property of the divergence, $\operatorname{div}_{\mu}(p X)=$ $p \operatorname{div}_{\mu}(X)+\mathcal{L}_{X} p$, and (6), this reduces to

$$
\begin{aligned}
\frac{d}{d t} p & =\frac{\partial p}{\partial t}+\operatorname{div}_{\mu}(p X)-p \operatorname{div}_{\mu}(X) \\
& =-p \operatorname{div}_{\mu}(X) .
\end{aligned}
$$

If the vector field $X$ is a Hamiltonian vector field on $(Q, \omega)$, the divergence vanishes, $\operatorname{div}_{\mu}(X)=0$ according to the Liouville theorem [29]. Therefore, the Fokker-Planck equation for a deterministic Hamiltonian system is represented by the following ordinary differential equation

$$
\frac{d}{d t} p=0
$$

This states that the probability density function is preserved along a Hamiltonian flow without stochastic diffusion effects. More precisely, (9) implies that the propagated probability density function at $t_{k+1}$ can be explicitly expressed as a composition of the backward flow map and the given probability density function at $t_{k}$

$$
p_{k+1}(R, \Omega)=p_{k}\left(\mathcal{F}^{-1}(R, \Omega)\right),
$$

where $\mathcal{F}: \mathrm{SO}(3) \times \mathbb{R}^{3} \rightarrow \mathrm{SO}(3) \times \mathbb{R}^{3}$ is the discrete flow map for the attitude dynamics. We can apply this equation recursively to propagate the probability density function over any time interval.

\section{B. Noncommutative Harmonic Analysis on $\mathrm{SO}(3)$}

Equation (10) provides a method to compute the probability density function at any time based on the probability density function at some prior time. As the flow is nonlinear, it is inefficient to characterize the density using its moments, since the moment expansion may decay slowly. Here, we propose a computational scheme that represents the probability density function for the attitude dynamics using noncommutative harmonic analysis on $\mathrm{SO}(3)$.

Noncommutative harmonic analysis is a generalization of Fourier analysis on Euclidean spaces to manifolds [4]. This is particularly useful since it provides a mathematical tool to approximate a probability density function based on samples of that function. More precisely, the propagated probability density function for the attitude dynamics of a rigid body can be expressed as

$$
p(R, \Omega)=\sum_{l=0}^{\infty} \frac{2 l+1}{(2 \pi)^{3}} \int_{\mathbb{R}^{3}} \exp (j \theta \cdot \Omega) \operatorname{tr}\left[P^{l}(\theta) U^{l}(R)\right] d \theta,
$$

where the vector $\theta \in \mathbb{R}^{3}$ and non-negative integer $l \in$ $\mathbb{N} \bigcup\{0\}$ are Fourier parameters, and the set of complex matrices $\left\{P^{l}(\theta) \in \mathbb{C}^{(2 l+1) \times(2 l+1)}\right\}_{l=0}^{\infty}$ is the Fourier spectrum of the density $p(R, \Omega)$. We denote the $l$-th irreducible unitary representation of $\mathrm{SO}(3)$ by $U^{l}(R) \in \mathbb{C}^{(2 l+1) \times(2 l+1)}$. A representation of a group is a homomorphism from the group to the set of invertible matrices, and by the Peter-Weyl theorem [30], the irreducible unitary representations form a complete orthonormal basis for the set of square-integrable functions on the group. The irreducible unitary representations can be expressed in various ways. For example, it can be expressed in terms of the wigner- $d$ functions using 3-1-3 Euler angles $\alpha, \beta, \gamma$ as

$$
U_{m, n}^{l}(R(\alpha, \beta, \gamma))=i^{m-n} e^{-i(m \alpha+n \gamma)} d_{m, n}^{l}(\cos \beta)
$$

for $-l \leq m, n \leq l$ [31]. The first few wigner- $d$ functions are given by

$$
\begin{aligned}
& d^{0}(\cos \beta)=1 \\
& d^{1}(\cos \beta)=\left[\begin{array}{lll}
\frac{1+\cos \beta}{2} & -\frac{\sin \beta}{\sqrt{2}} & \frac{1-\cos \beta}{2} \\
\frac{\sin \beta}{\sqrt{2}} & \cos \beta & -\frac{\sin \beta}{\sqrt{2}} \\
\frac{1-\cos \beta}{2} & \frac{\sin \beta}{\sqrt{2}} & \frac{1+\cos \beta}{2}
\end{array}\right] .
\end{aligned}
$$


Higher order wigner-d functions can be obtained using a recurrence relation [4].

From the orthnormal property of the irreducible unitary representation, the Fourier spectrum is computed by

$$
P^{l}(\theta)=\int_{\mathrm{SO}(3)} \int_{\mathbb{R}^{3}} p(R, \Omega) \exp (-j \theta \cdot \Omega) U^{l}\left(R^{-1}\right) d \Omega d R .
$$

The volume element for the rotation matrix $d R$ represents the Haar measure of $\mathrm{SO}(3)$; it can be written in terms of 3-1-3 Euler angles $\alpha, \beta, \gamma$ as $d R(\alpha, \beta, \gamma)=\frac{1}{8 \pi^{2}} \sin \beta d \alpha d \beta d \gamma$.

Substituting (10), we can compute the Fourier spectrum of the propagated probability density function. The propagated distribution can be reconstructed by (11). This is a global particle-based method to construct the propagated probability density function on $\mathrm{SO}(3) \times \mathbb{R}^{3}$.

\section{Visualization of the Attitude Uncertainty}

Let $p_{R}: \mathrm{SO}(3) \rightarrow \mathbb{R}$ be a probability density function on $\mathrm{SO}(3)$. For example, it can be obtained by integrating (11) over $\mathbb{R}^{3}$, i.e. $p_{R}(R)=\int_{\mathbb{R}^{3}} p(R, \Omega) d \Omega$. We propose a method for visualizing probability densities on $\mathrm{SO}(3)$ using three copies of two-spheres. The rotation matrix represents a linear transformation from a body fixed frame to an inertial frame. Therefore, the $i$-th column of the rotation matrix $R e_{i}$ represent the direction of the $i$-th body fixed axis in the inertial frame for $i \in\{1,2,3\}$. Since the vector $R e_{i}$ lies on the two-sphere $\mathbb{S}^{2}$, we can visualize uncertainties of $R e_{i}$ on a sphere either by color shading or by contour lines. Three copies of these spheres, one for each of the body fixed axes, can be used to visualize uncertainties on $\mathrm{SO}(3)$.

We find a marginal probability density of $p_{R}$ for each column of the rotation matrix. For given $r \in \mathbb{S}^{2}$, define a subset $H_{i}(r)$ of $\mathrm{SO}(3)$ as

$$
H_{i}(r)=\left\{R \in \mathrm{SO}(3) \mid R e_{i}=r\right\}
$$

for $i \in\{1,2,3\}$. This represents the set of rotation matrices whose $i$-th column is equal to the given direction $r$. Notice that $H_{i}(r)$ is invariant under the right action of a rotation about $e_{i}$, i.e., $H_{i}(r)=H_{i}(r) \exp S\left(\theta e_{i}\right)$, where $\theta \in \mathbb{S}^{1}$. As such, we can parameterize $H_{i}(r)$ in terms of $\theta \in \mathbb{S}^{1}$ and a representative element $R_{i}^{\circ}(r)$ of $H_{i}(r)$. More explicitly, we can choose $R_{i}^{\circ}(r)=\exp \left(\frac{\operatorname{acos}\left(r \cdot e_{i}\right)}{\left\|e_{i} \times r\right\|} S\left(e_{i} \times r\right)\right)$ if $e_{i} \times r \neq 0$. Then, the set $H_{i}(r)$ is parameterized as

$$
H_{i}(r)=\left\{R_{i}^{\circ}(r) \exp S\left(\theta e_{i}\right) \mid \theta \in \mathbb{S}^{1}\right\}
$$

The corresponding quotient space is the two-sphere, $\mathrm{SO}(3) / H_{i}(r) \simeq \mathbb{S}^{2}$. Using the properties of integration on a quotient space of a Lie group, we have

$$
\begin{aligned}
1 & =\int_{\mathrm{SO}(3)} p_{R}(R) d R \\
& =\int_{r \in S^{2}}\left(\frac{1}{2 \pi} \int_{\theta \in \mathbb{S}^{1}} p_{R}\left(R_{i}^{\circ}(r) \exp S\left(\theta e_{i}\right) d \theta\right) d r .\right.
\end{aligned}
$$

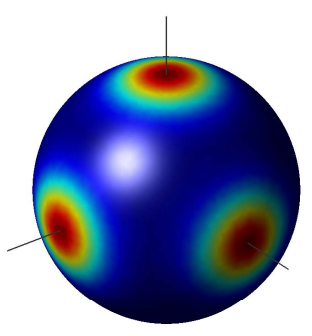

(a) Density 1

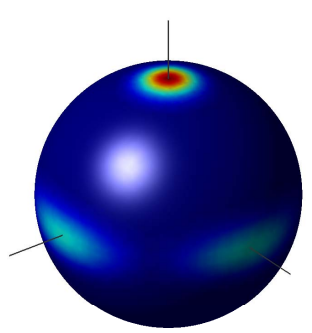

(c) Density 3

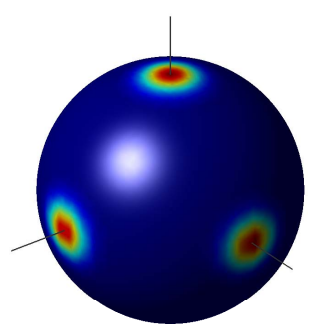

(b) Density 2

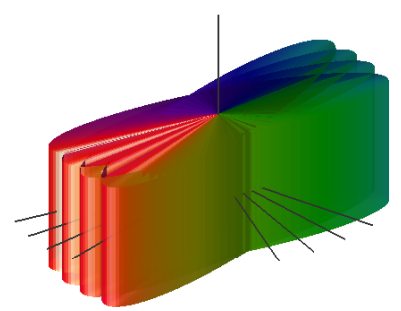

(d) Sample attitudes for the density 3
Fig. 1. Attitude uncertainty visualization example

Therefore, the marginal probability density for the $i$-th column of the rotation matrix, $p_{R}^{i}: \mathbb{S}^{2} \rightarrow \mathbb{R}$ is given by

$$
p_{R}^{i}(r)=\frac{1}{2 \pi} \int_{\theta \in \mathbb{S}^{1}} p_{R}\left(R_{i}^{\circ}(r) \exp \left(\theta \hat{e}_{i}\right)\right) d \theta .
$$

We plot these marginal probability density functions $p_{R}^{i}(r)$, that represent the probability density of the direction of the body fixed axes, on three two-spheres. If the magnitude of uncertainties is small, we can plot uncertainties for each body fixed axis on a single sphere, from which we can intuitively understand the attitude uncertainty of the rigid body.

Fig. 1 shows examples for the attitude probability density visualization. It is easily to see that the second density in Fig. 1(b) has smaller variation than the first density in Fig. 1(b). In the third density in Fig. 1(c), the vertical axis has smaller variation than the other horizontal axes. If we take a few samples from this density, we are likely to obtain the attitudes illustrated by Fig. 1(d), which reflect the fact that the direction of the vertical axis of the body in the inertial frame is well localized, but there is larger variation in the direction of other axes.

\section{Numerical COMPUTATIONS}

In this section, we propagate an initial probability density along the nontrivial dynamics of the 3D pendulum, and we visualize the attitude uncertainty. The properties of the pendulum are given by

$$
\begin{gathered}
J=\operatorname{diag}[0.13,0.28,0.17] \mathrm{kgm}^{2}, \quad m=1 \mathrm{~kg}, \\
\rho=0.3 e_{3} \mathrm{~m}, \quad g=9.81 \mathrm{~m} / \mathrm{s}^{2}
\end{gathered}
$$

The von Mises distribution (also known as the circular normal distribution) is a continuous probability density on 
the circle, which can be thought of as the circular analogue of the normal density [32].

$$
p(\theta)=\frac{1}{2 \pi I_{0}(\kappa)} \exp (\kappa \cos (\theta-\bar{\theta})),
$$

where $I_{0}$ is the zeroth order modified Bessel function of the first kind, given by $I_{0}(\kappa)=\sum_{i=0}^{\infty} \frac{\left(1 / 4 \kappa^{2}\right)^{i}}{(\kappa !)^{2}}$ with parameters $\kappa, \bar{\theta}$ that determine the shape of the density; as $\kappa$ increases, the density approaches a normal density with mean $\bar{\theta}$ and variance $\frac{1}{\kappa}$.

For two given rotation matrices $R, \bar{R} \in \mathrm{SO}(3)$, the quantity $\frac{1}{2}\left(\operatorname{tr}\left[\bar{R}^{T} R\right]-1\right)$ represents the cosine of the rotation angle between the two attitudes. Using this, we define a probability density function on $\mathrm{SO}(3)$ from the von Mises distribution. The probability distribution at the initial time is chosen as

$$
\begin{aligned}
p_{0}(R, \Omega) & =\frac{1}{c} \exp \left\{\frac{1}{2} \kappa\left(\operatorname{tr}\left[\bar{R}_{0}^{T} R\right]-1\right)\right\} \\
& \times \exp \left\{-\frac{1}{2}\left(\Omega-\bar{\Omega}_{0}\right)^{T} \Sigma^{-1}\left(\Omega-\bar{\Omega}_{0}\right)\right\},
\end{aligned}
$$

where $\bar{R}_{0}=I_{3 \times 3}, \bar{\Omega}_{0}=[4.14,4.14,4.14] \mathrm{rad} / \mathrm{s}, \Sigma=$ $0.1414^{2} I_{3 \times 3}$, and $\kappa=8$. The constant $c$ is a scaling factor chosen such that $\int p(R, \Omega) d R d \Omega=1$. The corresponding mean $\left(\bar{R}_{0}, \bar{\Omega}_{0}\right)$ yields an irregular, perhaps chaotic, attitude response [19].

We propagate this initial distribution using (10) and compute the Fourier spectrum using (13). The volume integration is approximated by Simpson's rule, and the flow map is computed using the Lie group variational integrator. The application of the Lie group variational integrator is particularly useful since it is symplectic, group structure preserving, and time reversible.

We have developed a parallel computing code using the MPI (Message Passing Interface) library, where the domain of integration is divided uniformly and distributed to each processor. This is desirable in terms of minimizing communication between processors and balancing the computational load among the processors. This algorithm has been implemented on 32 AMD Opeteron processors. Fig. 2 illustrates the propagated attitude uncertainty.

\section{Comments on Global Attitude Estimation}

The presented uncertainty propagation method can be applied to develop an attitude estimation scheme using Bayes rule. We first define a measurement model. We assume that the attitude and the angular velocity are measured by sensors to obtain

$$
z_{k}=\mathcal{Z}\left(R_{k}, \Omega_{k}\right)+v_{k}
$$

where $\mathcal{Z}: \mathrm{SO}(3) \times \mathbb{R}^{3} \rightarrow \mathbb{R}^{m}$ is a measurement function, $z_{k} \in \mathbb{R}^{m}$ is the measured value, and $v_{k} \in \mathbb{R}^{m}$ is measurement noise. For example, if we measure a direction to a known object $a \in \mathbb{S}^{2}$ and the angular velocity, the measurement function can be written as $\mathcal{Z}(R, \Omega)=\left[R^{T} a ; \Omega\right]$. We assume the probability density of the measurement noise $v_{k}$ is given by $p_{z_{k} \mid k}$, and it is an independent process.

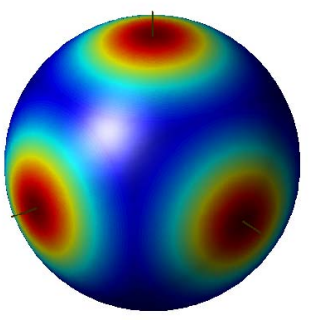

(a) $t=0.0$

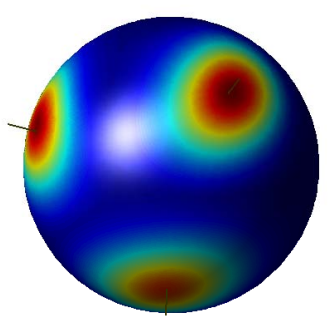

(c) $t=0.2$

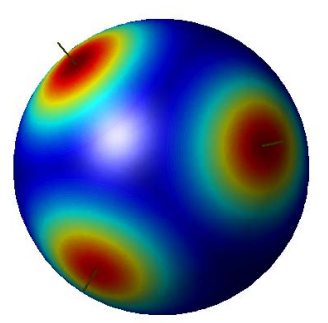

(b) $t=0.1$

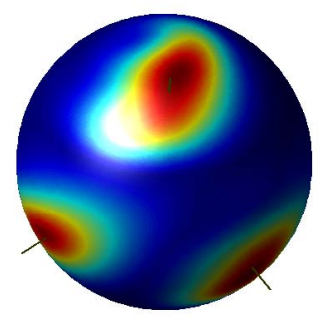

(d) $t=0.4$

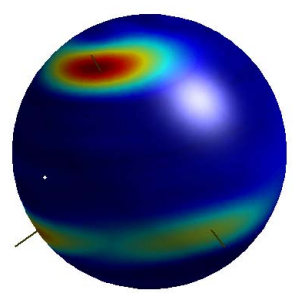

(e) $t=1.0$

Fig. 2. Propagated attitude uncertainty and max mean attitude

The set of all measurements from the initial time to $t_{k}$ is denoted by $Z_{k}=\left[z_{0}, z_{1}, \ldots, z_{k}\right]$. Suppose that we have a probability density function at the $k$-th time conditioned by $Z_{k}$, i.e., the expression for $p_{k \mid Z_{k}}$ is known, and a new measurement is obtained $z_{k+1}$ at $t_{k+1}$. Estimation can be described as finding an expression for $p_{k+1 \mid Z_{k+1}}$ given $p_{k \mid Z_{k}}$ and $Z_{k+1}=\left[Z_{k}, z_{k+1}\right]$. Using Bayes rule [33], we have

$$
\begin{aligned}
p_{k+1 \mid Z_{k+1}}(R, \Omega \mid[Z, z]) & \\
= & \frac{p_{z_{k+1} \mid k+1, Z_{k}}(z \mid R, \Omega, Z) p_{k+1 \mid Z_{k}}(R, \Omega \mid Z)}{p_{z_{k+1} \mid Z_{k}}(z \mid Z)} .
\end{aligned}
$$

Since the measurement processes are independent, we have $p_{z_{k+1} \mid k+1, Z_{k}}=p_{z_{k+1} \mid k+1}$, and the propagated density is given by (10), i.e., $p_{k+1 \mid Z_{k}}(R, \Omega \mid Z)=$ $p_{k \mid Z_{k}}\left(\mathcal{F}^{-1}(R, \Omega) \mid Z\right)$. The denominator is a normalizing constant that can be computed to satisfy $p_{z_{k+1} \mid Z_{k}}(z \mid Z)=$ $\int_{\mathrm{SO}(3) \times \mathbb{R}^{3}} p_{z_{k+1} \mid k+1}(R, \Omega) p_{k+1 \mid Z_{k}}(R, \Omega \mid Z) d R d \Omega$. In summary, the propagated probability density conditioned by the new measurement is given by

$$
\begin{aligned}
& p_{k+1 \mid Z_{k+1}}(R, \Omega \mid[Z, z]) \\
& \quad=\frac{1}{c} p_{z_{k+1} \mid k+1}(z \mid R, \Omega) p_{k \mid Z_{k}}\left(\mathcal{F}^{-1}(R, \Omega) \mid Z\right)
\end{aligned}
$$


for a normalizing constant $c$. This also can be represented using the harmonic analysis as in (11).

This expression is the key to solving the attitude estimation problem. This expression for $p_{x_{k+1} \mid Z_{k+1}}$ contains all the statistical information that can be derived from the attitude dynamics and the available measurements obtained up to the current time $t_{k+1}$. For example, using this probability density, we can compute the mean or the variance.

\section{CONCLUSIONS}

This paper addresses the problem of propagating the attitude uncertainty of a rigid body which evolves on the rotation group. The use of noncommutative harmonic analysis techniques to represent the uncertainty distribution in a global fashion overcomes a fundamental limitation of existing techniques, which implicitly assume that the uncertainty is localized or small. By exploiting the fact that the Liouville equation for a Hamiltonian system reduces to an ordinary differential equation, we are able to adopt a particle based approach for computing the advected probability density, thereby avoiding the computational expense of solving the equation as a numerical partial differential equation. By adopting a Lie group variational integrator as the underlying numerical scheme, we ensure that the resulting uncertainty propagation method inherits the geometric properties of the time evolution of probability densities in Hamiltonian systems, that arise from the symplectic geometry of the phase space.

A natural application of the proposed scheme is to the problem of global attitude estimation, particularly when the dynamics of the rigid body are extremely nonlinear, and the attitude measurements are relatively infrequent. Estimation problems with such characteristics are problematic for traditional techniques, such as the Kalman filter, which require frequent measurements, and relatively benign dynamics, in order to justify the localization and linearization assumptions built into the method.

\section{REFERENCES}

[1] J. Crassidis, F. Markley, and Y. Cheng, "Survey of nonlinear attitude estimation methods," Journal of Guidance, Control, and Dynamics, vol. 30 , no. 1 , pp. 12-28, 2007.

[2] E. Lefferts, F. Markley, and M. Shuster, "Complementary filter design on the special orthogonal group $\mathrm{SO}(3)$," in Proceedings of the AIAA Aerospace Sciences Meeting, 1982, AIAA-1982-70.

[3] R. Mahony, T. Hamel, and J. Pflimlin, "Complementary filter design on the special orthogonal group $\mathrm{SO}(3)$," in Proceedings of the IEEE Conference on Decision and Control, 2005, pp. 1477-1484.

[4] G. Chirikjian and A. Kyatkin, Engineering applications of noncommutative harmonic analysis. Boca Raton, FL: CRC Press, 2001.

[5] D. Xiu, "Efficient collocational approach for parametric uncertainty analysis," Commun. Comput. Phys., vol. 2, no. 2, pp. 293-309, 2007.

[6] D. Xiu and J. Hesthaven, "High-order collocation methods for differential equations with random inputs," SIAM J. Sci. Comput., vol. 27, no. 3, pp. 1118-1139 (electronic), 2005.

[7] E. Hairer, C. Lubich, and G. Wanner, Geometric Numerical Integration, 2nd ed., ser. Springer Series in Computational Mathematics. Springer-Verlag, 2006, vol. 31.

[8] P. Diaconis, Group Representations in Probability and Statistics. Institute of Mathematical Statistics, 1988.

[9] K. Elworthy, Stochastic Differential Equations on Manifolds. Cambridge University Press, 1982.

[10] M. Emery, Stochastic Calculus in Manifolds. Springer, 1989.
[11] J. Lo and L. Eshleman, "Exponential Fourier densities on SO(3) and optimal estimation and detection for rotational processes," SIAM Journal on Applied Mathematics, vol. 36, no. 1, pp. 73-82, 1979.

[12] H. Hendriks, "Nonparametric estimation of a probability density on a Riemannian manifold using Fourier expansions," The Annals of Statistics, vol. 18 , no. 2, pp. 832-849, 1990.

[13] P. Kim, "Deconvolution density estimation on $\mathrm{SO}(\mathrm{n})$," The Annals of Statistics, vol. 26, no. 3, pp. 1083-1102, 1998.

[14] W. Park, J. Kim, Y. Zhou, N. Cowan, A. Okamura, and G. Chirikjian, "Diffusion-based motion planning for a nonholonomic flexible needle model," in Proceedings of the 2005 IEEE International Conference on Robotics and Automation, 2005.

[15] Y. Wang, Y. Zhou, D. Maslen, and G. Chirikjian, "Solving phase-noise Fokker-Planck equations using the motion-group Fourier transform," IEEE Transactions on Communications, vol. 54, no. 5, pp. 868-877, 2006.

[16] F. Markley, "Attitude filtering on SO(3)," in Proceedings of the AAS Malcolm D. Shuster Astronautics Symposium, 2005, AAS 05-460.

[17] D. Scheeres, F. Hsiao, R. Park, B. Villac, and J. Maruskin, "Fundamental limits on spacecraft orbit uncertainty and distribution propagation," in Proceedings of the AAS Malcolm D. Shuster Astronautics Symposium, 2005, AAS 05-471.

[18] J. Valpiani and P. Palmer, "Nonliner symplectic attitude estimation for small satellitese," in Proceedings of the AIAA/AAS Astrodynamics Specialist Conference and Exhibit, 2006, AIAA 2006-6159.

[19] T. Lee, N. Chaturvedi, A. Sanyal, M. Leok, and N. H. McClamroch, "Propagation of uncertainty in rigid body attitude flows," in Proceedings of the IEEE Conference on Decision and Control, 2007, pp. 26892694.

[20] T. Lee, M. Leok, and N. H. McClamroch, "Lie group variational integrators for the full body problem," Computer Methods in Applied Mechanics and Engineering, vol. 196, pp. 2907-2924, May 2007.

[21] — "Lie group variational integrators for the full body problem in orbital mechanics," Celestial Mechanics and Dynamical Astronomy, vol. 98, no. 2, pp. 121-144, June 2007.

[22] M. Gromov, "Pseudo holomorphic curves in symplectic manifolds," Invent. Math., vol. 82, no. 2, pp. 307-347, 1985.

[23] F. Hsiao and D. J.Scheeres, "Fundamental constraints on uncertainty evolution in Hamiltonian systems," IEEE Transactions on Automatic Control, vol. 52, no. 4, pp. 686-691, 2007.

[24] J. Lazaro-Cami and J. Ortega, "Stochastic Hamiltonian dynamical systems," 2007. [Online]. Available: http://www.citebase.org/abstract?id=oai:arXiv.org:math/0702787

[25] N. Bou-Rabee and H. Owhadi, "Stochastic variational integrators," 2007. [Online]. Available: http://www.citebase.org/abstract?id=oai:arXiv.org:0708.2187

[26] L. Wang, "Variational integrators and generating functions for stochastic hamiltonian systems," Ph.D. dissertation, Universität Karlsruhe, 2007.

[27] J. Shen, A. Sanyal, N. Chaturvedi, D. Bernstein, and N. McClamroch, "Dynamics and control of a 3D pendulum," Proceedings of the IEEE Conference on Decision and Control, pp. 323-328, 2004.

[28] T. Lee, "Computational geometric mechanics and control of rigid bodies," Ph.D. dissertation, University of Michigan, 2008.

[29] J. Marsden and T. Ratiu, Introduction to Mechanics and Symmetry. Springer, 1999.

[30] F. Peter and H. Weyl, "Die vollständigkeit der primitiven darstellungen einer geschlossenen kontinuierlichen gruppe," Math. Ann., vol. 97, pp. 735-755, 1927.

[31] L. Biedenharn and J. Louck, Angular Momentum in Quantum Physics. Addison-Wesley, 1981.

[32] von Mises, "Über die Ganzzahligkeit der Atomgewicht und verwandte Fragen," Physikal, Z, vol. 19, pp. 490-500, 1918.

[33] A. Jazwinski, Stochastic Process and Filtering Theory. Academic Press, 1970. 